Tohoku J. exp. Med., 1973, 109, 173-187

\title{
Interaction between the Splenic and Hepatic Arteries in Portal Hypertension
}

\author{
Hidemi Yamauchi, Kyojr Yamamoto,* Kenji Koyama \\ Yasushi Matsuo, Seitchi Kashimura and Tosio Sato \\ The First Department of Surgery, Tohoku University School \\ of Medicine, Sendai
}

\begin{abstract}
Yamadoh, H. Yamamoto, K., Koyama, K., Matsuo, Y., Kashomura, S. and Sato, T. Interaction between the Splenic and Hepatic Arteries in Portal Hypertension. Tohoku J. exp. Med., 1973, 109 (2), 173-187—Experimental production of an arterio-venous fistula between the splenic artery and vein of the dog causes increased splenic arterial flow, and reduction of hepatic arterial flow. In the majority of clinical cases of splenomegaly of periportal fibrosis (Banti's syndrome), selective celiac angiography reveals narrowing of the hepatic artery proper, and there is a significant negative correlation between the radii of splenic and hepatic arteries on the $x$-ray films. The results suggest reduced hepatic arterial flow in such cases. Two different mechanisms are taken into consideration for the explanation of this circulatory condition: There may be some hemodynamical interaction between the portal system and the hepatic artery, or increased blood flow of the splenic artery, or increased blood flow of the splenic artery may reduce the hepatic share of celiac arterial flow. No arteriographical narrowing of the hepatio artery proper was demonstrated in splenomegalic eirrhosis even if high portal inflow is expected through the massive splenomegaly. A comparison of the radius of the hepatic artery proper and the percentage of intrahepatic shunted flow supports the view that the effective portal flow is unchanged in those cases. —_ splenic artery; hepatic artery; portal flow
\end{abstract}

Dilatation of the splenic artery in splenomegalic disorders is especially pronounced in cases of portal hypertension. In previous reports of ours (Yamauchi 1968; Yamauchi et al. 1970) a remarkable increase in the radii of the splenic arteries in periportal fibrosis accompanied by increased blood flow and concomitant narrowing of the proper hepatic artery was arteriographically or histometrically demonstrated. The purpose of this paper is to examine possible interaction between the splenic and hepatic arterial systems in the clinical and experimental portal hypertension.

\section{Materials and Methods}

\section{Clinical investigations}

Measurement of splenic blood flow: Splenic blood flow was measured at laparotomy by means of the dye dilution technique (Koyama 1967) or a square wave electromagnetic flowmeter (Nihonkoden MF-5) in $\mathbf{1 4}$ eases of periportal fibrosis, 6 cases of splenomegalic

Received for publication, July 25, 1972.

* Present address: Department of Surgery, Mito Kyodo Hospital, Mito. 
cirrhosis and 12 cases of splenomegaly without portal hypertension, consisting of 10 cases of congenital hemolytic jaundice, one case of idiopathic thrombocytopenic purpura and one case of myeloma.

Estimation of the radii of the splenic and hepatic arteries, and portal and splenic veins: The radii of the arteries on the films of selective celiac angiography were estimated in 30 cases of controls, 22 cases of poriportal fibrosis, 14 cases of splenomegalic cirrhosis, 7 cases of cirrhosis and 7 cases of splenomegaly without portal hypertension. The estimation was done in patients without hepatopetal shunts from the superior mesenteric arteries. The estimated values were corrected by the diameter of the catheter $(2.4 \mathrm{~mm})$. On the other hand, if the mean radius of the splenic or portal vein trunk on the films of the splenoportography performed in these eases is $\mathrm{d}$, the diameter of a puncture needle used for the splenoportography is $\mathrm{n}$ and its diameter in the films is $\mathrm{N}$, the estimated radius of the splenic or portal vein in the living body is then given as $D(m m)=d \cdot n / 2 N$. The values of $n / N$ varies little among all the examined cases, and are approximately equal to 1/1.3. Accordingly, we obtain $\mathrm{D}(\mathrm{mm})=\mathrm{d} / 2.6$. The measurements were made 3 times each at the portal vein trunk and the hilum of the spleen.

\section{Experimental studies}

Direct measurement of arterial blood flow and arterial radius: Fifteen mongrel dogs weighing approximately $17-24 \mathrm{~kg}$ were anesthetized by intravenous injection of sodium pentobarbital at an initial dose of $30 \mathrm{mg} / \mathrm{kg}$. All the dogs received intubation and were kept under respiratory control with a mechanical ventilator. The femoral artery cannulation was carried out and the pressure was recorded continuously using a transducer and an electronic manometer. After laparotomy non-cannulating flow probes were positioned around the femoral, splenic, common hepatic, renal, superior mesenteric, left gastric and iliac arteries and connected to a flowmeter. The nerve plexus was carefully stripped parallel to the long axis and preserved intact in each case. The flow soon after the application of the probes was variable, but it was fairly stabilized by the end of approximately thirty minutes, when the recordings were taken. Temporary downstream occlusion of each artery rendered zero flow base line. To every dog heparin sodium was administered at an initial dose of approximately $100 \mathrm{u} / \mathrm{kg}$ and $500 \mathrm{ml}$ of dextran was given intravenously by drip. After the estimation of blood flow each vessel was cut transversely, and a vascular ring of five millimeter in length was excised at the site of the measurement, and fixed with formalin for 24 hours. After fixation the ring was opened and stretched flat for the estimation of vascular radius.

Experimental model of portal hypertension: Seven mongrel dogs weighing $20-24 \mathrm{~kg}$ were laparotomized through a midline abdominal incision. A polyethylene tube filled with heparinized saline was introduced into the portal vein trunk via a branch of the superior mesenteric vein. Portal pressure was measured with a water manometer. The splenic artery had been exposed free and a flow probe connected to the flowmeter was placed on it. An effort was made to ligate all collateral vessels around the spleen. Silica particle suspension was injected into the intrahepatic portal vein branches, and the changes in portal pressure and splenic blood flow were recorded. The splenic artery was cut, and a cannula of $2 \mathrm{~mm}$ in diameter was inserted into it, while another one with a diameter of 5 $\mathrm{mm}$ was placed into the splenic vein with its tip toward the portal vein trunk. The two cannulae were connected to each other by means of a siliconized vinyl tube with a diameter of $10 \mathrm{~mm}$, in which a flow probe was set. A shunted pathway between the splenic artery (SA) and the splenic vein (SV) was thus created (SA-SV shunt) (Fig. 1). The dogs with acute portal vein obstruction by silica particles and SA-SV shunt were used as the models of portal hypertension, because they had many characteristics common to those of the clinical cases of periportal fibrosis with high portal pressure, presinusoidal block and an increase in splenic arterial flow following reduced splenie resistance. The influence of portal pressure on splenic artery flow was further ascertained in these models. 


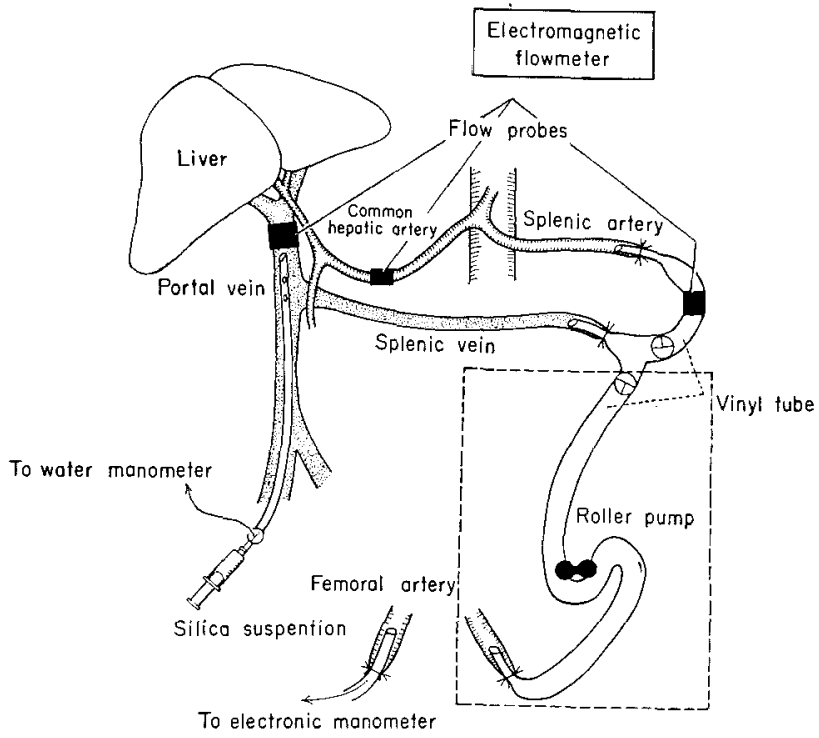

Fig. 1. An experimental model of acute portal hypertension. Silica suspension was infused into the portal vein branches in order to produce presinusoidal block. The production of a fistula between the splenic artery and splenic vein (SA-SV shunt) causes a high portal vein inflow. A supplementary circuit (dotted line) was added to the model in an experimental animal to accelerate portal flow artificially using a roller pump without increasing splenic arterial flow.

The effect of varying splenic arterial flow on hepatic arterial flow: The experiments were performed on four mongrel dogs weighing $17-25 \mathrm{~kg}$. During laparotomy common hepatic artery flow was measured with a flowmeter. In two of them the gastroduodenal artery had been ligated. The changes in common hepatic artery flow immediately after the clamping or releasing the splenic artery were recorded. Similar attempts were mado in the animals after the creation of the SA-SV shunt with or without the injection of silica suspension.

The influence of augmentation of portal flow through the splenic vein on hepatic artery flow: In one of these 4 dogs a supplementary circuit was produced (Fig. 1, dotted line); namely, a fistula between the splenic vein and the femoral artery was created by the use of a siliconized vinyl tube. A roller pump was placed in the circuit. One round per minute of the pump produced a flow of $6 \mathrm{ml} / \mathrm{min}$. Flow probes were positioned around the portal vein and the hepatic artery and connected to the flowmeters. The influence of an increase in splenic vein flow with a roller pump on hepatic arterial flow, portal flow, portal pressure and arterial pressure before and after the injection of silica particles were assessed simultaneousily. The experimental procedure was repeated three to four times to obtain reliable information.

The effect of an SA-FV shunt on hepatic artery flow: In another dog, a shunt between the splenic artery and the femoral vein (SA-FV shunt) was produced with a vinyl tube and the effect of clamp or release of the shunted route on hepatic arterial flow was examined. 


\section{Results}

Studies in clinical cases

The results of splenic arterial flow in various disorders are summarised in Tables 1 and 2, together with portal pressure and the radii of the splenic artery and vein. In Fig. 2, the radius of the splenic arteries in the control and various disorders is plotted on the abscissa of a double logarithmic coordinate system against splenic arterial flow measured with either dye dilution technique or an

TABLE 1. Splenic arterial flow by means of dye dilution technique

\begin{tabular}{r|c|c|l|c|c|c|c}
\hline Case & Age & Sex & \multicolumn{1}{|c|}{ Diagnosis } & $\begin{array}{c}\text { Portal } \\
\text { pressure } \\
\left(\mathrm{cmH}_{2} \mathrm{O}\right)\end{array}$ & $\begin{array}{c}\text { Splenic } \\
\text { arterial } \\
\text { flow } \\
(\mathrm{ml} / \mathrm{min})\end{array}$ & $\begin{array}{c}\text { Radius of } \\
\text { splenic } \\
\text { artery } \\
\text { (mm) }\end{array}$ & $\begin{array}{c}\text { Radius of } \\
\text { splenic } \\
\text { vein } \\
(\mathrm{mm})\end{array}$ \\
\hline 1 & 62 & $\mathrm{f}$ & Periportal fibrosis & 26 & 730 & 2.9 & 4.8 \\
2 & 63 & $\mathrm{f}$ & Periportal fibrosis & 38 & 1039 & 3.2 & 6.6 \\
3 & 39 & $\mathrm{~m}$ & Periportal fibrosis & 37 & 692 & 3.0 & 5.0 \\
4 & 41 & $\mathrm{f}$ & Splenomegalic cirrhosis & 33 & 691 & 2.8 & 4.7 \\
5 & 28 & $\mathrm{f}$ & Periportal fibrosis & 19 & 548 & 2.7 & 5.0 \\
6 & 33 & $\mathrm{f}$ & Periportal fibrosis & 24 & 976 & 3.5 & 6.2 \\
7 & 23 & $\mathrm{~m}$ & Splenomegalic cirrhosis & 38 & 1260 & 3.8 & 8.9 \\
8 & 41 & $\mathrm{~m}$ & Splenomegalic cirrhosis & 29 & 710 & 2.9 & 5.4 \\
9 & 56 & $\mathrm{f}$ & Periportal fibrosis & 34 & 1820 & 4.1 & 6.0 \\
10 & 16 & $\mathrm{f}$ & CH. & 14.5 & 300 & 2.3 & 3.2 \\
11 & 23 & $\mathrm{~m}$ & CHJ & 14.5 & 669 & 3.0 & 4.3 \\
12 & 52 & $\mathrm{f}$ & Plasmacytoma & & 450 & 2.8 & \\
13 & 26 & $\mathrm{f}$ & TTP* & 15 & 438 & 2.5 & 3.5 \\
14 & 28 & $\mathrm{f}$ & CHJ & 14 & 850 & 4.0 & 6.0 \\
15 & 36 & $\mathrm{f}$ & CHJ & 14 & 420 & 2.8 & 3.9 \\
16 & 32 & $\mathrm{~m}$ & CHJ & 11 & 516 & 3.2 &
\end{tabular}

* CHJ, congenital hemolytic jaundice.

* ITP, idiopathic thrombocytopenic purpura.

TABLE 2. Splenic arterial flow by the use of an electromagnetic flowmeter

\begin{tabular}{|c|c|c|c|c|c|c|c|}
\hline Case & Age & Sex & Diagnosis & $\begin{array}{c}\text { Portal } \\
\text { pressure } \\
\left(\mathrm{cmH}_{2} \mathrm{O}\right)\end{array}$ & $\begin{array}{c}\text { Splenic } \\
\text { arterial } \\
\text { flow } \\
(\mathrm{ml} / \mathrm{min})\end{array}$ & $\begin{array}{l}\text { Radius of } \\
\text { splenic } \\
\text { artery } \\
(\mathrm{mm})\end{array}$ & $\begin{array}{l}\text { Radius of } \\
\text { splenic } \\
\text { vein } \\
(\mathrm{mm})\end{array}$ \\
\hline 1 & 51 & $\mathrm{~m}$ & Periportal fibrosis & 30 & 480 & 3.0 & 4.8 \\
\hline 2 & 25 & f & Periportal fibrosis & 24 & 600 & 3.6 & 7.4 \\
\hline 3 & 41 & f & Periportal fibrosis & 27 & 350 & 2.7 & 5.8 \\
\hline 4 & 46 & f & Periportal fibrosis & 37 & 450 & 3.5 & 5.5 \\
\hline 5 & 66 & $\mathrm{~m}$ & Splenomegalic cirrhosis & 26 & 600 & 3.3 & 5.9 \\
\hline 6 & 38 & $\mathrm{~m}$ & Splenomegalic cirrhosis & 35 & 700 & 3.8 & 6.9 \\
\hline 7 & 35 & f & Periportal fibrosis & 44 & 580 & 3.0 & 5.8 \\
\hline 8 & 67 & f & Periportal fibrosis & 38 & 750 & 3.4 & 5.8 \\
\hline 9 & 43 & f & Periportal fibrosis & 32 & 580 & 3.4 & 6.1 \\
\hline 10 & 50 & f & Periportal fibrosis & 35 & 200 & 3.3 & \\
\hline 11 & 43 & $\mathrm{~m}$ & Splenomegalic cirrhosis & 33 & 200 & 3.0 & 5.6 \\
\hline 12 & 28 & f & CHJ & 13 & 800 & 4.0 & 6.0 \\
\hline 13 & 21 & $f$ & ITP & & 420 & $\begin{array}{l}3.2 \\
3.2\end{array}$ & 5.7 \\
\hline 14 & 26 & f & CHJ & 14 & 180 & 2.8 & 3.9 \\
\hline 15 & 32 & f & CHJ & 13 & 200 & 2.9 & 4.3 \\
\hline 16 & 23 & $\mathrm{~m}$ & CHJ & 15 & 600 & 3.2 & 5.1 \\
\hline
\end{tabular}



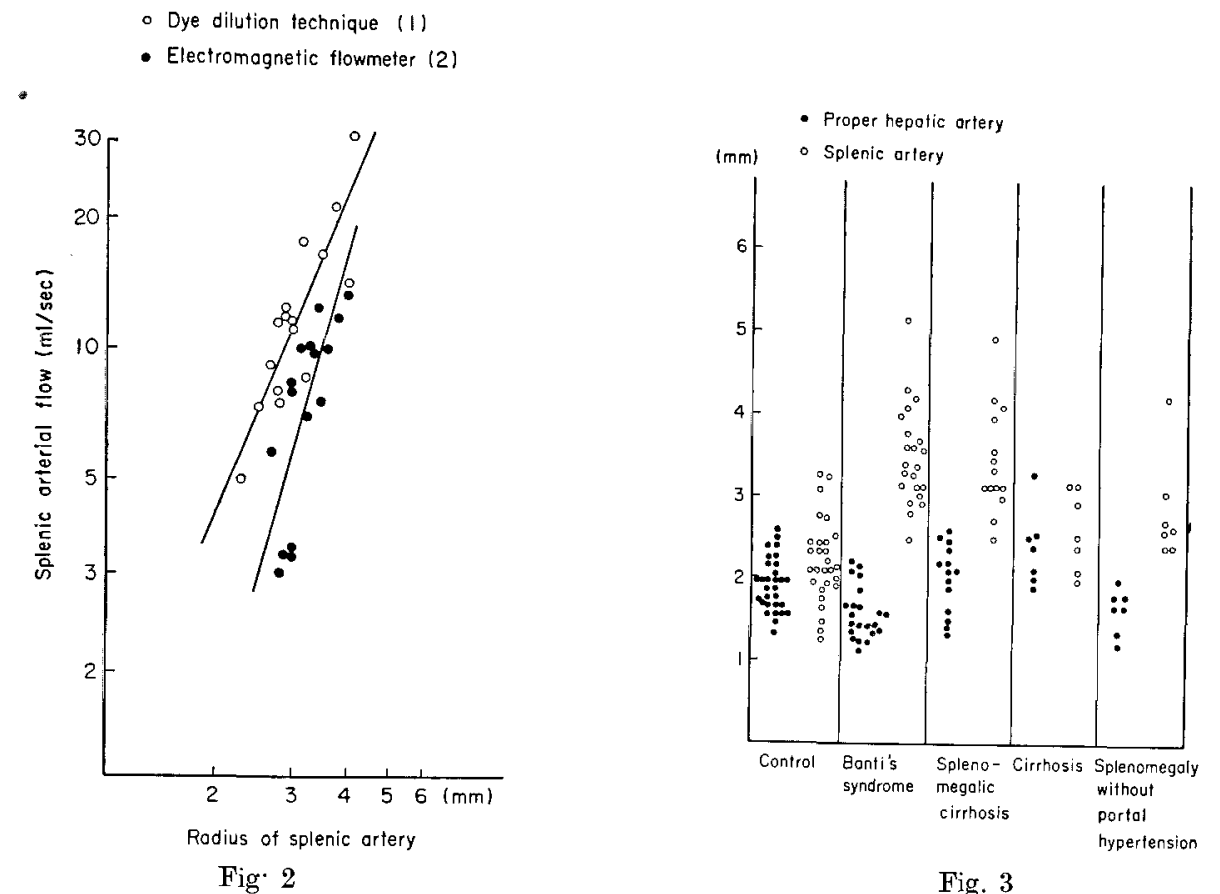

Fig. 2. Relationship between splenic arterial flow and the radii of the splenic arteries. A close correlation is ascertained. Regression equations, correlation coefficients and their levels of significance are:

1) $\mathrm{Y}=2.43 \mathrm{X}-0.12, \mathrm{r}=0.86, \mathrm{p}<0.01$.

2) $\mathrm{Y}=3.57 \mathrm{X}-0.95, \mathrm{r}=0.76, \mathrm{p}<0.01$.

Fig. 3. Estimated values of the radii of the splenic and hepatic arteries proper. Note narrowing of the hepatic arteries proper in Banti's syndrome and dilatation of the splenic arteries in splenomegalic cirrhosis and Banti's syndrome.

electromagnetic flowmeter on the ordinate. Although the values obtained from the flowmeter vary considerably, linear correlations are found in the estimates in each group, the correlation coefficient being 0.86 or 0.76 , which is statistically significant at $1 \%$ level. The value estimated by the dilution technique at a certain radius of the splenic artery is markedly higher than that by the flowmeter. The calculated value of the radius of the hepatic artery proper and the splenic artery in control, periportal fibrosis, splenomegalic cirrhosis, usual cirrhosis and splenomegaly without portal hypertension are shown in Fig. 3 ; the mean and standard deviation are $1.83 \pm 0.34(\mathrm{~mm}), 1.53 \pm 0.29,2.00 \pm 0.35,2.33 \pm 0.42$ and $1.57 \pm 0.22$, respectively. The estimates in the cases of periportal fibrosis and splenomegaly without portal hypertension are significantly smaller than the control $(p<0.01)$. No difference in the radii of these vessels was found between the cases of splenomegalic cirrhosis and the control group. However, the vessels in usual cirrhosis are significantly dilated $(\mathrm{p}<0.01)$.

The splenic arterial flow significantly correlates to the radius of the splenic vein $(\mathrm{p}<0.01$, Fig. 4). 
A significant correlation of the radii of the splenic artery and vein in the control and those of splenomegaly without portal hypertension or in those of periportal fibrosis and splenomegalic cirrhosis was ascertained, with a correlation coefficient of 0.72 or 0.79 (Fig. 5). Between the slopes of (1) and (2), a significant difference is noted.

The estimated values of the radii of the trunk of the portal and splenic veins are demonstrated in Fig. 6, where distinct elevation is found in portal hypertension.

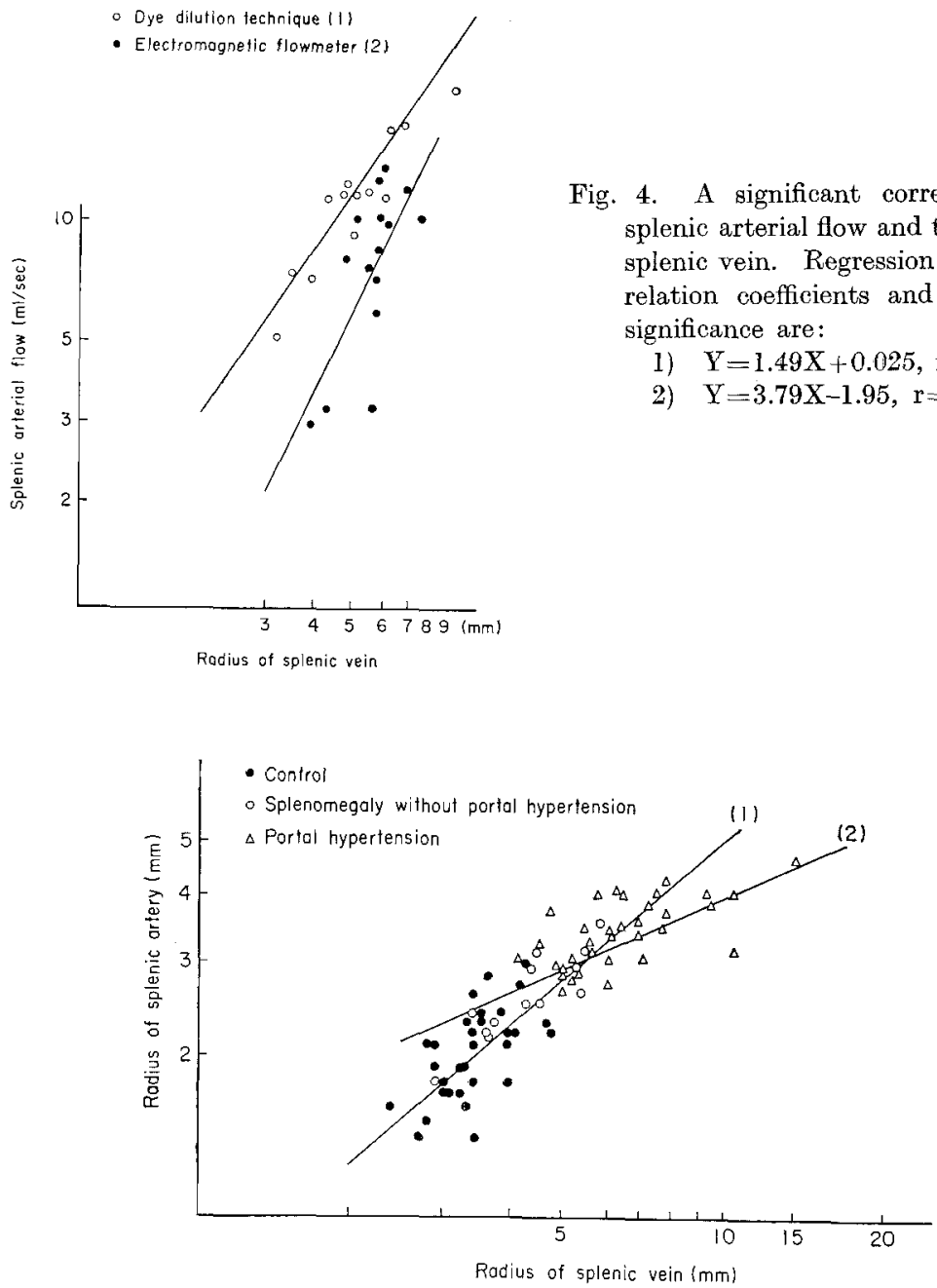

Fig. 5. A significant correlation is found between the radius of the splenic artery and vein. Regression equations, correlation coefficients and their levels of significance are:

1) $Y=0.86 X-0.15, \mathrm{r}=0.80, \mathrm{p}<0.01$.

2) $\mathrm{Y}=0.43 \mathrm{X}+0.19, \mathrm{r}=0.19, \mathrm{p}<0.01$. 


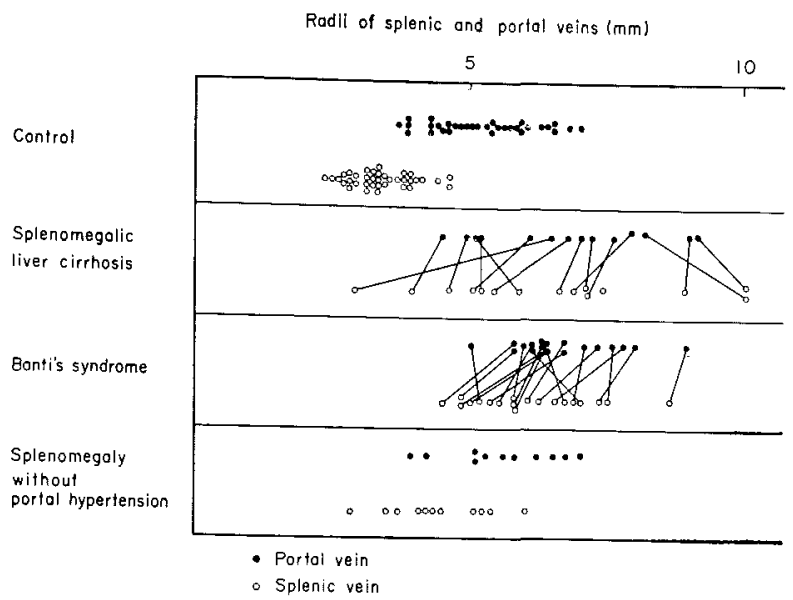

Fig. 6. The calculated estimates of the radii of the splenic and portal veins. Pronounced dilatation of the splenic and portal veins in the cases of portal hypertension is noted.

\section{Experimental studies}

As is shown in Fig. 7, a highly significant correlation was found between the arterial radius and arterial blood flow in the dogs as well as in the clinical cases.

On the other hand, a sequential decrease in splenic arterial flow was illustrated corresponding to progressive elevation of portal pressure (Fig. 8). When the portal pressure was held within $300-400 \mathrm{~mm} \mathrm{H} \mathrm{H}_{2} \mathrm{O}$, the arterial pressure remained approximately at a value from 80 to $100 \mathrm{~mm} \mathrm{Hg}$ throughout the course of the experiment until a shock state developed.

Fig. 7. Correlation between the arterial blood flow in the experimental dogs and arterial radius. A relationship quite similar to that in the clinical cases is demonstrated. Regression equation, correlation coefficient and its level of significance are: $\mathrm{Y}=2.79 \mathrm{X}-6.71, \mathrm{x}=0.80, \mathrm{p}<0.01$.

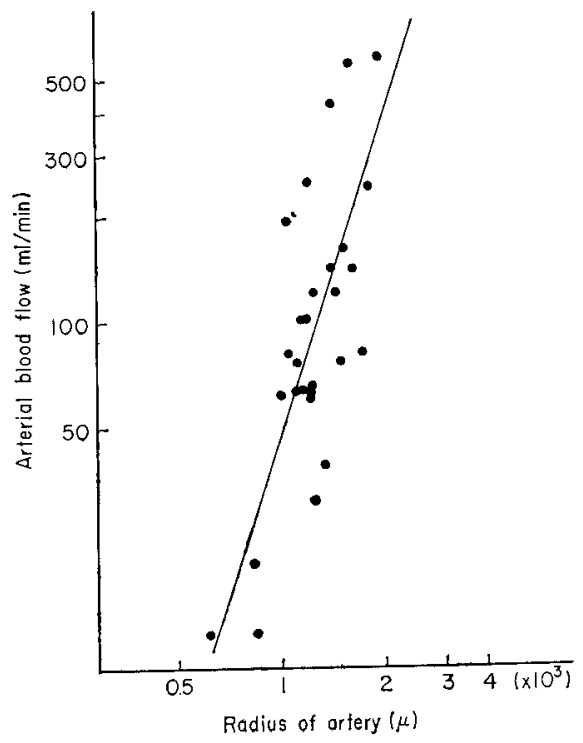




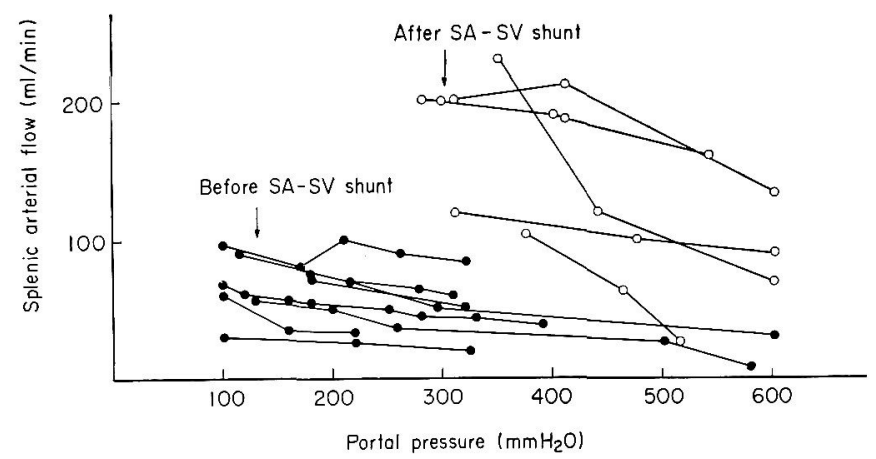

Fig. 8. Changes in splenic arterial flow in experimental portal hypertension. Decline in splenic artery flow following the progressive elevation of portal pressure. After the production of an SA-SV shunt splenic arterial flow is increased 2 to 3 times.

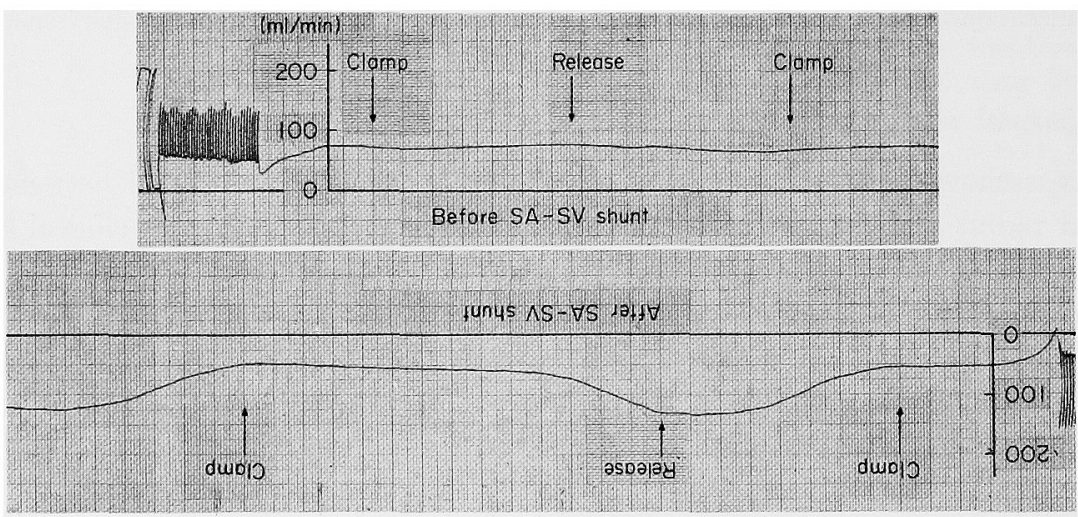

Fig. 9. Changes in hepatic arterial flow following acute variations in splenic arterial flow by the clamping or releasing of the splenic artery. The magnitude of the changes is remarkably enhanced after the production of an SA-SV shunt.

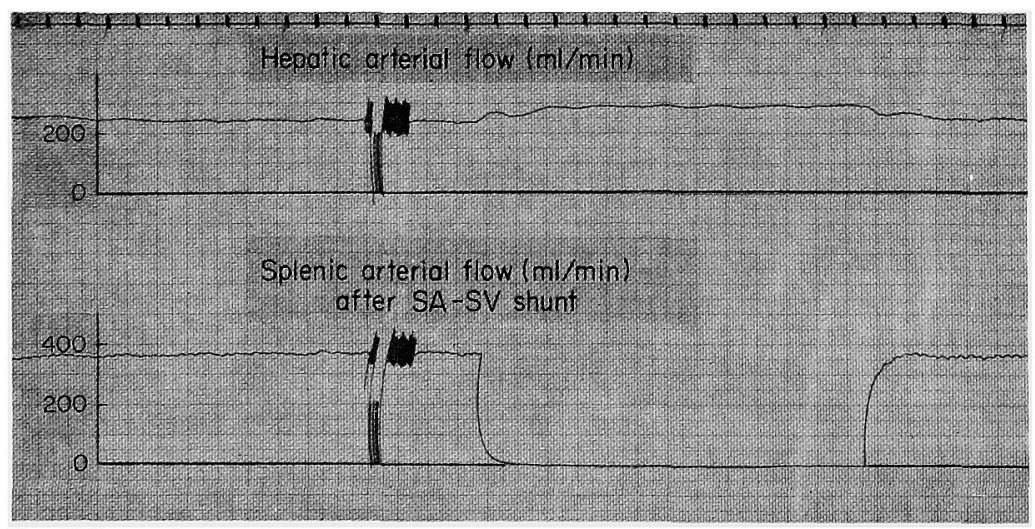

Fig. 10. Effect of acute variation in splenic arterial flow after the production of an SASV shunt on hepatic arterial flow. 
The increase or decrease in hepatic arterial flow of $6-25 \%$ was noted after clamping or releasing the splenic artery in control animals (Fig. 9). This tendency was more pronounced in dogs with an SA-SV shunt or in the experimental model of portal hypertension (Figs. 10 and 11). If the "increase rate" is defined as percentage of the rise in hepatic artery flow after the splenic arterial clamping of the hepatic artery flow after releasing the clamp, and "decrese rate" as that of the fall in hepatic artery flow after releasing the clamp of the hepatic arterial flow after the splenic arterial clamping, the values of the former and the latter respectively become gradually higher and lower as the splenic arterial flow is increased regardless of the kind of the experimental procedures (Fig. 12).

An artificial increase in portal flow through the splenic vein by the use of a



Fig. 11. Effect of acute diversion in splenic arterial flow after the production of an SA-SV shunt in an experimental model of portal hypertension on hepatic arterial flow.

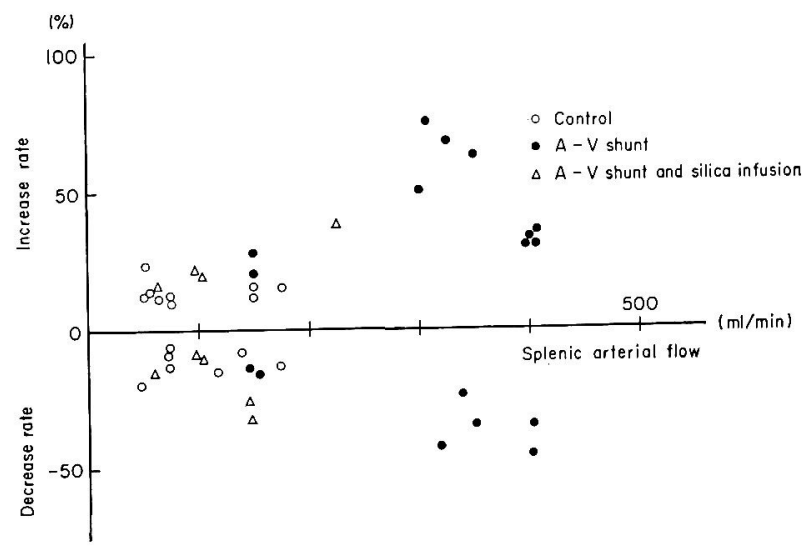

Fig. 12. Relationship between the increasing or decreasing rates in hepatic and splenic arterial flows. Note the decline in the decrease rate corresponding to a graded increase in splenic arterial flow (for details see text). 


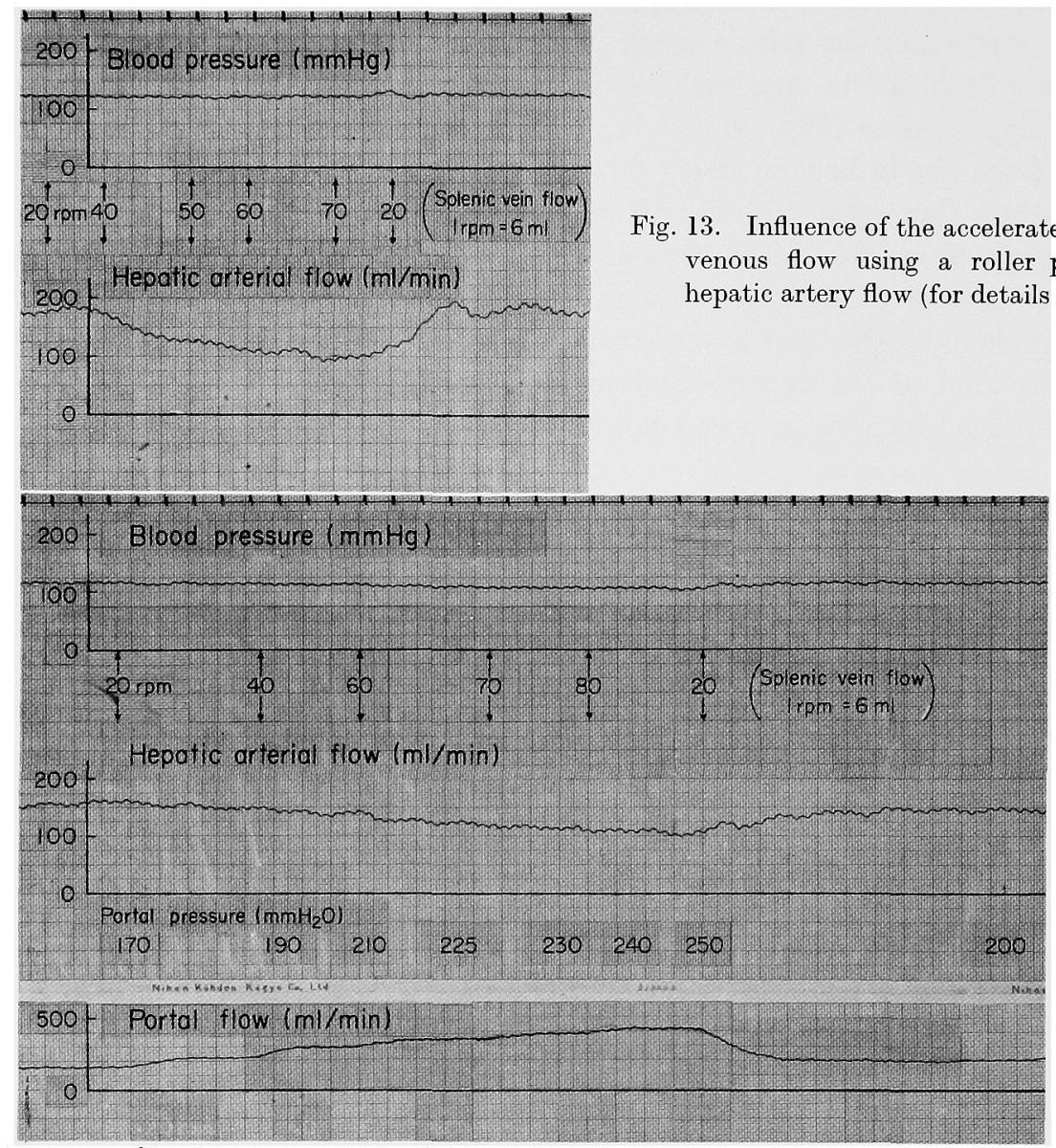

Fig. 14. Simultaneous record of blood pressure, hepatic arterial flow, portal pressure and portal flow corresponding to an artificial increase in splenic venous flow in the experimental model of portal hypertension. Hepatic arterial flow is declined as portal flow is increased in steps without noticeable changes in blood pressure.

roller pump induced a graded decrease in hepatic arterial flow in the model of portal hypertension as well as in control dogs (Figs. 13 and 14). The simultaneous records of hepatic and splenic arterial flows after the production of the SA-SV shunt are presented in Fig. 15. A distinct decrease in hepatic arterial flow after producing the shunt is observed.

\section{Discussion}

Angiometry and venometry

A quite close correlation between the radius of the splenic arterial trunk and splenic arterial flow determined by both the dye dilution technique and the flowmeter during operation was reported previously (Koyama et al. 1970). A consider- 


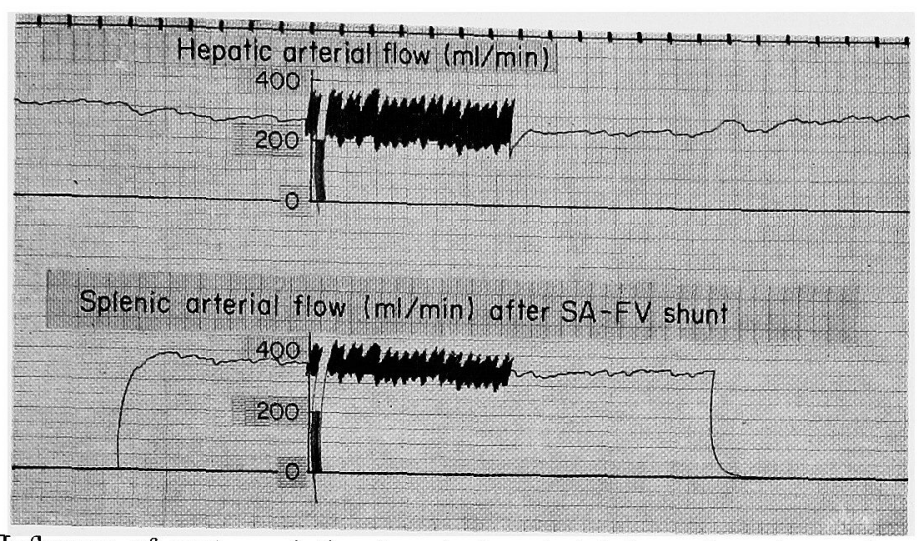

Fig. 15. Influence of acute variation in splenic arterial flow after the production of the splenie artery (SA) to femoral vein (FV) shunt (SA-FV shunt). A ppreciable decrease in hepatic arterial flow after the release of the splenic arterial block 2 is is demonstrated.

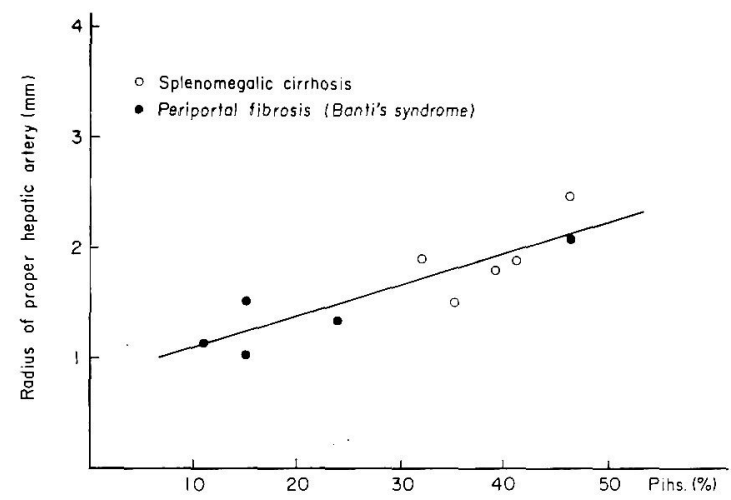

Fig. 16. A significant correlation between the percentag $ə$ of intrahspatic shunted blood flow (PIHS) and the radius of the hepatic artery proper in the cases of periportal fibrosis and splenomegalic cirrhosis. Regression equation, correlation coefficient and its level of significance are:

$$
\mathrm{Y}=0.03 \mathrm{X}+0.85, \mathrm{r}=0.85, \mathrm{p}<0.01 \text {. }
$$

able difference was demonstrated between the absolute values obtained from the flowmeter and those by dye dilution technique. However, the significant correlation with the arterial radius was found in both groups. We confirmed the relationship also in the experimental animals. It seems consequently justified to assume a reduction in hepatic arterial flow on the basis of arteriographically demonstrable narrowing of the hepatic artery.

The radius of the splenic vein is distinctly proportional to that of the splenic artery on the double logarithmic coordinate system in controls as well as in cases of splenomegaly with or without portal hypertension. However, the regression coefficients are different according to whether there is portal hypertension or not. 
In the cases of portal hypertension, dilatation of the splenic vein is more pronounced than in the controls and the cases of splenomegaly without portal hypertension. The stronger dilatation is possibly due to venous congestion. Although there is a significant correlation between splenic arterial flow and the radius of the splenic vein, the velocity of blood stream in the venous system seems to be variable. If extrahepatic shunted flow through the spleen is small, it may be concluded that the splenic vein is gradually dilated as the splenic artery grows and splenic venous flow is increased even in portal hypertension.

\section{Acute experimental model of portal hypertension}

Reduction in the splenic arterial flow following temporary occulusion of the splenic vein was already pointed out by Womack and Peters (1961). In the present study a remarkable decrease in the splenic arterial flow was demonstrated after the injection of silica particles into the portal vein branches. Recently, however, an experimental evidence to the contrary was presented using some ion-exchange resin (Gotoh 1971): namely, a rise in the splenic arterial flow was possible despite portal hypertension. Some other factors should be taken into account for explaining this peculiar finding. It appears obvious in the experimental model of portal hypertension that an increase in splenic arterial flow to twice or three times that of the control was possible against high portal pressure as is usually observed in clinical cases of portal hypertension. This result indicates reduced resistance to splenic circulation in clinical cases of sustained high portal pressure.

\section{Portal inflow in the cases of portal hypertension}

Portal flow is decreased in acute experimental portal hypertension (Makiguchi 1966) and in relatively large number of patients with cirrhosis (Bradley et al. 1952; Moreno et al. 1967). However, in "splenomegalic portal hypertension" an increase in portal flow through the large spleen is expected. Viamonte et al. (1970) maintained that increased portal flow in cirrhosis was caused by hemodynamically significant splanchnic arteriovenous shunting. It was assumed by some authors (Boyer et al. 1967; Ueda et al. 1969; Isomatsu et al. 1971) that an increase in portal flow through an enormous splenomegaly might play an important role in portal hypertension in patients with periportal fibrosis. Another view was held by Nakamura and Nakamura (1969) that a rise in the portal flow was insignificant on account of the development of extrahepatic shunted routes. A measurement of portal blood flow in splenomegalic cirrhosis and periportal fibrosis was actually carried out by Gotoh (1971), who reported slightly increased values in comparison with the controls. A significant correlation between the radius of the splenic vein and splenic arterial flow, a close one between the radius of the splenic artery and vein and marked dilatation of the portal vein in some of the cases of splenomegalic portal hypertension, cirrhosis or non-cirrhotic periportal fibrosis, suggest high blood flow of the portal vein. 
Interaction between the splenic and hepatic artery

It will be accepted from the above-mentioned result that an increase in splenic artery flow causes a decrease in hepatic arterial flow in the dogs with or without portal hypertension. Two possible explanations on the mechanism are as follows.

Firstly, when one of the two branches from an arterial trunk receives reduced blood flow, a decrease in blood flow takes place in the other. This process is visualized by experimental evidence that the creation of a shunted pathway between the splenic artery and femoral vein results in a distinct reduction in hepatic arterial flow.

Secondly, an increased portal flow through an enlarged spleen gives rise to a decrease in hepatic arterial flow by way of some interaction between the hepatic arterial and portal venous systems. Since Burton-Opitz (1911) demonstrated the interaction using a flowmeter, a number of experimental and clinical works have been reported (Bauer et al. 1932; Sancetta 1953; Condon et al. 1962; Schenk et al. 1962; and Hanson and Johnson 1966).

Discussions have been focussed on the influence of a transient reduction in portal flow on the hepatic arterial flow in cases of intact animals. There have been few publications conscerning the influence of increased portal flow upon the hepatic arterial flow through the splenic vein in situ. Experimentally, hepatic arterial flow can be reduced by augmentation of the portal flow through the splenic vein by the use of a roller pump. It is therefore reasonable to presume that an increase in splenic arterial flow is one of the causing factors in decreasing hepatic arterial flow.

The possible mechanism of the interaction between portal venous and hepatic arterial systems still remains obscure. Bauer et al. (1932) could demonstrate that a reduction in hepatic arterial flow was followed by an acceleration of portal venous flow in their perfusion experiment with the animal liver and assumed that the influence of portal circulation on the hepatic artery is exerted through pressure in the lobular sinusoids. Hanson and Johnson (1966) introduced a hypothesis that myogenic constriction of hepatic arterial resistance vessels is triggered by increase in the pressure in the sinusoid and plays a significant role in a common mechanism of autoregulation of hepatic arterial flow and arterial response to elevated venous pressure and to portal blood flow.

The narrowing of the hepatic artery proper in periportal fibrosis associated with reduction of hepatic arterial flow is regarded as the result of the augmented splenic blood flow possibly with an increase in portal flow. Because the radius of the hepatic artery proper remains within the normal values in splenomegalic cirrhosis, the portal flow is also probably in the normal range on account of the interaction between the hepatic artery and portal vein systems. However, in most of the cases of splenomegalc cirrhosis, increased flow of the portal system is expected from remarkable dilatation of the splenic artery and it seems likely that a decrease in the hepatic arterial flow is caused, which may be aggravated by mechanical arterial obstruction 
on account of periportal fibrosis. In spite of such a hemodynamical condition, no angiographical narrowing of the hepatic artery proper was actually demonstrated, and the reason of this incongruity is still obscure. However, some difference in the quantity of intrahepatic shunted blood flow (PIHS), which was demonstrated by Nakamura and Nakamura (1969) in this group, may be responsible for the difference in the hemodynamics. The mean and standard deviation of PIHS in 9 cases of periportal fibrosis and 6 cases of splenomegalic cirrhosis are $20 \pm 11.5(\%)$ and $43 \pm 11.7$ $(\%)$, respectively. The difference was statistically significant $(\mathrm{p}<0.01)$. Moreover, it is of interest to find a statistically highly significant correlation between the radius of the hepatic artery and the value of PIHS ( $p<0.01$ ) (Fig. 16). It is concluded that in splenomegalic cirrhosis the "effective" portal flow is unchanged by the development of the intrahepatic shunts, even if high portal inflow is present on account of marked increase in splenic arterial flow. One of the patients with portal fibrosis with a high estimate of PIHS of $46 \%$ exhibited exceptionally a dilatation of the hepatic artery proper as is shown in cirrhosis. In such a case a response of the hepatic artery similar to that in decreased portal vein perfusion seems to take place.

No tendency toward a decrease in proper hepatic arterial flow was observed in splenomegalic cirrhosis in general. However, narrowed hepatic arteries proper were found in some cases; a rise in portal flow is assumed in such cases, because the patients exhibit a hyperkinetic syndrome similar to Banti's syndrome.

Ueda et al. (1968) previously reported angiographically demonstrable narrowing of the hepatic artery proper and high portal inflow by means of the scintigram in Banti's syndrome. Our present study supports their view in part, but exact measurement of the radius on the films reveals a narrowing of the proper hepatic artery also in about half the cases of splenomegalic cirrhosis. Therefore, the narrowing of the hepatic artery is by no means specific for periportal fibrosis.

In usual cirrhosis without a typical splenomegaly, a significant dilatation of the hepatic artery was demonstrated compared with the controls and splenomegalic cirrhosis. As a number of reports have confirmed reduced portal flow in cirrhosis, increased hepatic arterial flow may be assumed on the ground of the interaction between the portal and hepatic arterial systems.

In congenital hemolytic jaundice with a marked splenomegaly, hepatic arterial flow appears to be decreased with a possible increase in portal flow. Some influence of the age on the arterial development is considered in these cases.

It is also of diagnostic value that the radius of the hepatic artery proper is smaller than $2 \mathrm{~mm}$ in almost all the cases of periportal fibrosis or in about half the cases of splenomegalic cirrhosis and more than $2 \mathrm{~mm}$ in the majority of usual cirrhosis.

\section{References}

1) Bauer, W., Dale, H.H., Poulsson, L.T. \& Richards, D.W. (1932) The control of circulation through the liver. J. Physiol., 74, 343-375. 
2) Boyer, J.L., Sen Gupta, K.P., Biswas, S.K., Pal, N.C. \& Basu Mallick, K.C. (1967) Idiopathic portal hypertension. Ann. intern. Med., 66, 41-68.

3) Bradley, S.E., Ingelfinger, F.J. \& Bradley, G.P. (1952) Hepatic circulation in cirrhosis of the liver. Circulation., 5, 419-429.

4) Burton-Opitz, R. (1911) The vascularity of the liver. II. The influence of the portal blood-flow upon the flow in the hepatic artery. Quart. J. exp. Physiol., 4, 93-102.

5) Condon, R.E., Nyphus, L.M., Chapman, N.D., \& Harkins H.N. (1962) Portal vein and hepatic artery interaction: Studies in the isolated, perfused liver. Gastroenterology, 43, 547-556.

6) Gotoh, H. (1971) Studies on the splenic circulation in portal hypertension using trapezoidal-wave electromagnetic flowmeter. Jap. J. Gastroent. (Jap.), 68, 103-122.

7) Hanson, K.M. \& Johnson, P.C. (1966) Local control of hepatic arterial and portal venous flow in the dog. Amer. J. Physiol, 211, 712-720.

8) Isomatsu, T., Takase, H., Tsukada, M., Azumi, T., Atsuda, T., Hayasaka, S., Shiroishi, T., Itoh, S., Samejima, N. \& Sugie, S. (1971) Significance of increased portal flow. Nippon Geka Gakkai Zasshi (Jap.)., 72, 1303-1305.

9) Koyama, K. (1967) Hemodynamics of the spleen in Banti's dyndrome. Tohoku J. exp. Med., 93, 199-217.

10) Koyama, K., Hamauchi, H., Watanabe, K., Kimura, S., Kashimura, S. \& Sato, T. (1970) Hemodynamies of the spleen and the liver viewed from arteriographic studies. Geka (Jap.), 32, 1033-1040.

11) Makiguchi, Y. (1966) Studies on hemodynamics in portal hypertension. Jap. J. Gastroent. (Jap.), 63, 33-47.

12) Moreno, A.H., Burchell, A.R., Rousselot, L.M., Panke, W.P., Slafsky, S.F. \& Burke, J.H. (1967) Portal blood flow in cirrhosis of the liver. J. clin. Invest., 46, 436445.

13) Nakamura, T. \& Nakamura, S. (1969) Organ characteristics on vascular system in man. 2. Vascular characteristics of the liver. Tohoku Igaku Zasshi (Jap.), 77, I80255.

14) Sancetta, S.M. (1953) Dynamic and neurogenic factors determining the hepatic arterial flow after portal occlusion. Circulat. Res., 1, 414418.

15) Sato, T., Kakizaki, G., Suda, Y. \& Saito, Y. (1965) On the pathogenesis of Banti's syndrome. Tohoku $J$. exp. Med., 83, 375-390.

16) Schenk, W.G., McDonald, J.C., McDonald, K.\& Drapanas, T. (1962) Direct measurement of hepatic blood flow in surgical patients: With related observations on hepatic blood flow dynamics in experimental animals. Ann. Surg., 156, 463-471.

17) Ueda, H., Kitani, K., Kameda, H., Nagatani, M., Chiba, K., Takeda, T., Iio, M. \& Yamada, H. (1969) Significance of splenic blood flow in portal hypertension. Saishin Igaku (Jap.), 24, 1740-1749.

18) Viamonte, M., Danner, P., Warren, W.D. \& Fomon, J. (1970) A new technique for the assessment of hyperkinetic portal hypertension. Radiology, 96, 539-542.

19) Womack, N.A. \& Peters, R.M. (1961) The significance of splenomegaly in cirrhosis of the liver. Ann. Surg., 153, $1006-1019$.

20) Yamauchi, H. (1968) Estimation of blood flow in the Banti spleen on anatomical basis. Tohoku J. exp. Med., 95, 63-77.

21) Yamauchi, H., Suda, Y., Yamamoto, K. \& Sato, T. (1970) Arteriographic studies of splenic and hepatic arteries in portal hypertension. Tohoku J.exp. Med., 101, 363374. 